\title{
Supporting Information: Indirect Interlayer Bonding in Graphene-Topological Insulator Van der Waals Heterostructure: Giant Spin-Orbit Splitting of the Graphene Dirac States
}

\author{
Shivani Rajput, ${ }^{*}$ Yao-Yi Li, Michael Weinert, and Lian Li \\ Department of Physics, University of Wisconsin, Milwaukee, Wisconsin 53201, USA \\ E-mail: srajput@uwm.edu
}

\section{$k$-projected Graphene-Bi $\mathrm{Se}_{3}$ bands}

The bands $k$-projected to the graphene cell (and weighted by the spatial localization on the graphene) for a graphene- $\mathrm{Bi}_{2} \mathrm{Se}_{3}$ separation of $3.875 \AA$ and the registry shown in Fig. S1(b) are given in Fig. $\mathrm{S} 1(\mathrm{c})$. The graphene $\mathrm{K}$ and $\mathrm{K}^{\prime}$ points correspond to $\Gamma$ of different zones and also different directions in the $\mathrm{Bi}_{2} \mathrm{Se}_{3} \mathrm{BZ}$. The general features of the bands show one set of bands with a degeneracy at the Dirac point and another set of upward and downward dispersing bands split away from the Dirac point. There are, however, noticeable differences in the dispersion that may be observable in angle resolved photoemission spectroscopy. Most notably, the intensity between $\mathrm{K}$ and $\mathrm{K}^{\prime}$ and along the different directions vary with momentum and between the inner/outer edges of the spin-orbit split bands, which reflect the breaking of the graphene sublattice symmetry and a Rashba-type splitting. For probes that integrate for $k$-space, such as STS, or for multiple orientations, adding the $\mathrm{K}$ and $\mathrm{K}^{\prime}$ contributions should provide the more direct comparison.

The possibility of a proximity induced effect on the graphene bands can already be anticipated by considering the overlap of the valence electrons of graphene and $\mathrm{Bi}_{2} \mathrm{Se}_{3}$, Fig. $\mathrm{S} 1(\mathrm{~d})$,

\footnotetext{
*To whom correspondence should be addressed
}
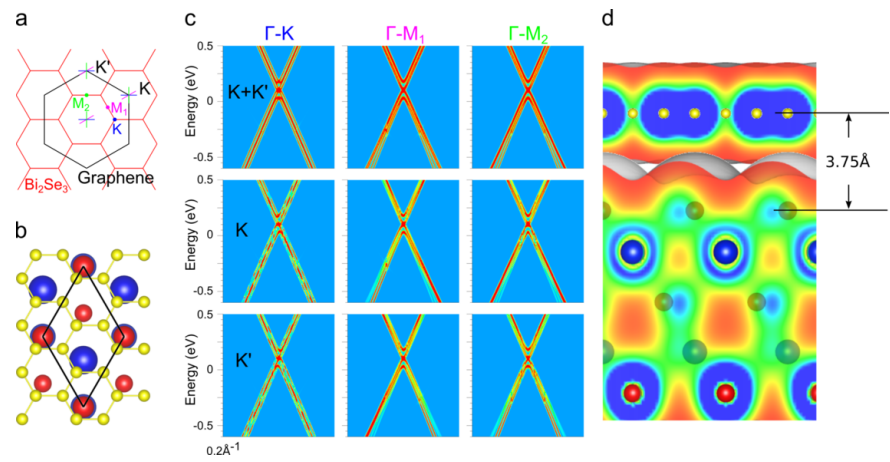

Figure S1: (a) Brillouin zones for $\mathrm{Bi}_{2} \mathrm{Se}_{3}$ and graphene, with the high symmetry points for each marked. The graphene $\mathrm{K}$ and $\mathrm{K}^{\prime}$ points fold back to the $\Gamma$ point of the $\mathrm{Bi}_{2} \mathrm{Se}_{3} \mathrm{BZ}$. The colored lines show different cuts along high symmetry directions around $\Gamma$ and the $\mathrm{K}$, $\mathrm{K}^{\prime}$. (b) Ball-and-stick model of graphene on $\mathrm{Bi}_{2} \mathrm{Se}_{3}(0001)$ with a $\sqrt{3} \times \sqrt{3}$ epitaxial relationship. Yellow ball: carbon; blue ball: Bi; red ball: Se. (c) Calculated bands $k$-projected onto the $(1 \times 1)$ graphene BZ around $\mathrm{K}$ and $\mathrm{K}^{\prime}$ along different directions for graphene/1QL $\mathrm{Bi}_{2} \mathrm{Se}_{3}$ for a separation of $3.875 \AA$. The colors correspond to the calculated relative momentum-resolved weight of the states. (d) Cross-sectional view of the overlap of valence densities of separated graphene and $\mathrm{Bi}_{2} \mathrm{Se}_{3}$. The isosurface is $10^{-3}$ $e^{-} / a_{B}^{3}$ and the maximum of the R-G-B color on the planar cuts is $0.05 e^{-} / a_{B}^{3}$. 
which is small but still significant at a separation of $\sim 3.75 \AA$. This indicates that proximity induced effects are expected, though with a strong dependence on separation due to the exponential decay of this density in the interface region.

\section{Calculated bands at the interface of the graphene/ $\mathrm{Bi}_{2} \mathrm{Se}_{3}$}

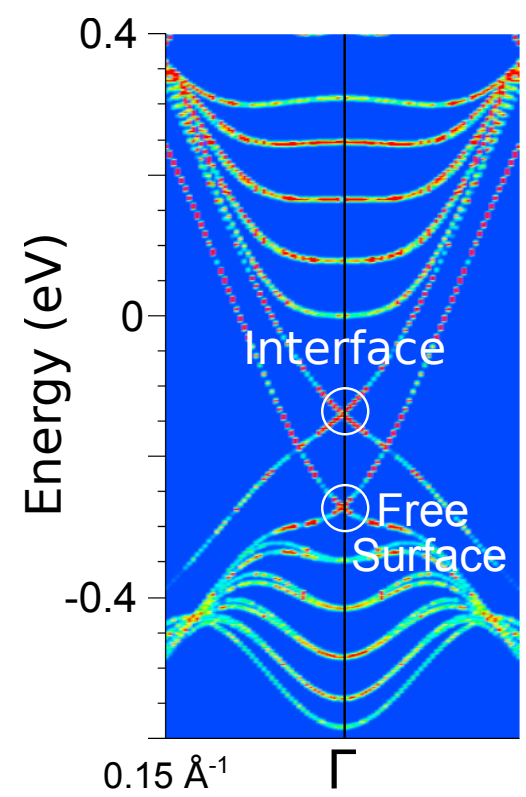

Figure S2: Calculated bands at the interface QL of the $7 \mathrm{QL} \mathrm{Bi}_{2} \mathrm{Se}_{3}+$ graphene at a separation of $3.875 \AA$. The topological Dirac states at the interface is shifted towards the Fermi level compared to that from the free surface.

As discussed in the main text, the spin-orbit induced splitting and topology of the graphene bands are the same for both the single QL $\mathrm{Bi}_{2} \mathrm{Se}_{3}$ substrate - which is too thin to support the $\mathrm{Bi}_{2} \mathrm{Se}_{3}$ TI Dirac state - and the $7 \mathrm{QL}$ one. As shown here, Fig. S2, however, the interaction of the graphene and the Bi2Se3 does shift the position of the 7 QL TI Dirac state towards the middle of the gap and now localized at the interface.

\section{Spatial distribution of Graphene and $\mathrm{Bi}_{2} \mathrm{Se}_{3}$ Dirac States}

Overlayer and substrate states will most interact strongly when they overlap both spatially and energetically (assuming compatible symmetries). In the calculations, the TI and graphene Dirac states are distinguishable energetically, and are shown in Fig. S3. The TI surface states, Fig. S3(a), have significant weight on the Se. On the other hand, Fig. S3(b), which is the same as Fig. 1(d) but for a smaller value of the isosurface, shows that the density in the plane of the topmost Se atoms is effectively zero. These differences in spatial distribution imply that the direct interaction of the substrate and graphene Dirac states should be small. a

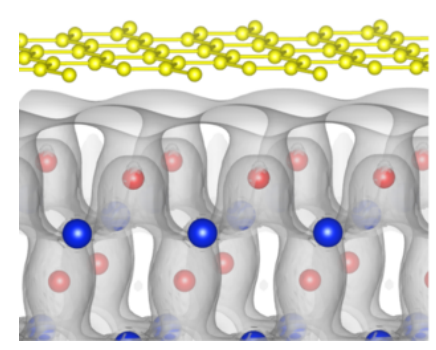

b

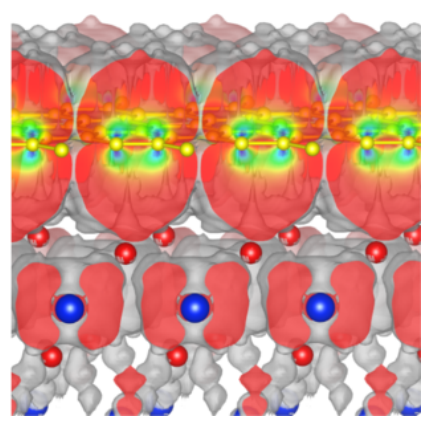

Figure S3: (a) Isosurface $\left(5 \times 10^{-5} e^{\left.-/ a_{B}^{3}\right)}\right.$ of the $1 \mathrm{QL} \mathrm{Bi}_{2} \mathrm{Se}_{3}$ surface state that evolves into the TI Dirac state. Note that this state is mainly on the Se atoms. Yellow ball: carbon; blue ball: $\mathrm{Bi}$; red ball: Se. (b) Density corresponding to the graphene Dirac point states for the graphene $/ \mathrm{Bi}_{2} \mathrm{Se}_{3}$ junction, showing that graphene $\pi$ orbitals also have weight on the $\mathrm{Bi}$ atoms, which accounts for the large transferred spin-orbit splitting. (Isosurface: $2.5 \times 10^{-5} e^{-} / a_{B}^{3}$; maximum of the $\mathrm{R}-\mathrm{G}-\mathrm{B}$ color is $0.125 e^{-} / a_{B}^{3}$.) 


\section{Impact of water exposure on $\mathrm{Bi}_{2} \mathrm{Se}_{3}$ Dirac states.}

Since the $\mathrm{Bi}_{2} \mathrm{Se}_{3}$ film was exposed to deionized (DI) water during the graphene transfer process, control experiments were carried out to investigate the effect of water on the electronic properties of the TI film. $\mathrm{Bi}_{2} \mathrm{Se}_{3}$ film was removed from UHV, dipped into DI water for 60 sec and re-introduced back into UHV for STM and STS study. STM imaging suggests no substantial effect on surface morphology of $\mathrm{Bi}_{2} \mathrm{Se}_{3}$, Fig. S4(a). While the line shape of the $\mathrm{dI} / \mathrm{dV}$ spectra remains unchanged, the Dirac point now appears at $-425 \mathrm{meV}$, Fig. S4(b). Compared to as-grown $\mathrm{Bi}_{2} \mathrm{Se}_{3}$ films, this indicates a shift of $\sim 175 \mathrm{meV}$ away from the Fermi level, suggesting that water exposure further electron dopes the $\mathrm{Bi}_{2} \mathrm{Se}_{3}$ film. Nevertheless, the Dirac surface states remain intact, consistent with angle resolved photoemission spectroscopy study of adsorption of water molecules in UHV. ${ }^{1}$
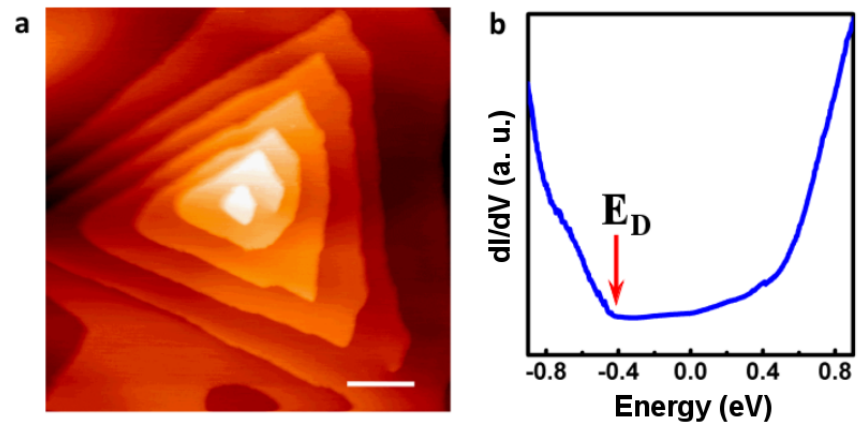

Figure S4: (a) STM image of a $30 \mathrm{~nm}$ MBEgrown $\mathrm{Bi}_{2} \mathrm{Se}_{3}$ film after dipping in water for one minute $\left(I_{t}=0.1 \mathrm{nA}, V_{s}=1.02 \mathrm{~V}, 50 \mathrm{~nm}\right.$ scale bar). (b) $d I / d V$ spectrum taken on the waterexposed $\mathrm{Bi}_{2} \mathrm{Se}_{3}$ film, showing the shift of $\mathrm{E}_{\mathrm{D}}$ away from the Fermi level.

\section{Calculated Graphene Density of States}

As discussed in the text, spin-orbit and broken inversion symmetry split the graphene Dirac state into a cone at $\mathrm{E}_{\mathrm{D}}$ plus two spin-orbit split states that have quadratic dispersion near the band edges and then (approximately) linear dispersion slightly away. The density of states

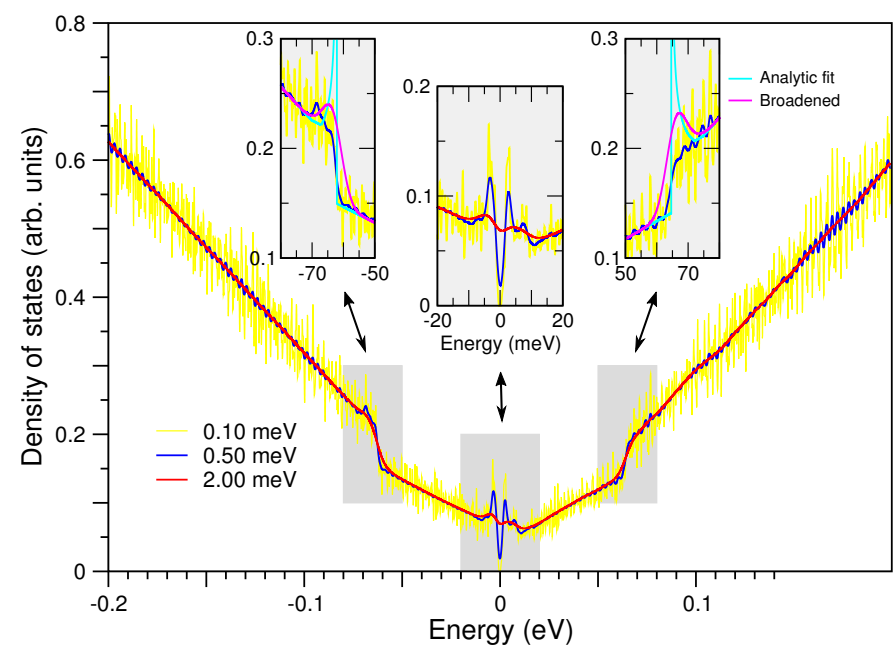

Figure S5: Calculated density of states around the gaps for the laterally shifted graphene $/ \mathrm{Bi}_{2} \mathrm{Se}_{3}$ at a separation of $3.875 \AA$. The calculations were carried out on a fine mesh (with $\sim 150,000$ eigenvalues in the energy and $k$. The insets give expanded views of the DOS near the band edges. For the smallest broadening $(0.1 \mathrm{meV}=1.1 \mathrm{~K})$, two peaks on either side of the gap are seen containing the states pushed out of the gap. As the thermal broadening increases from $0.1 \mathrm{meV}$ (yellow) to $2.0 \mathrm{meV}$ ( $23 \mathrm{~K}$, red), the peaks and gap quickly disappear, leaving the $\mathrm{V}$-shape DOS near $\mathrm{E}_{\mathrm{D}}$ at LN2 temperature.

(DOS) should thus be $\mathrm{V}$-shaped around $\mathrm{E}_{\mathrm{D}}$, show jumps at the energies corresponding to the spin-orbit split states (due to the quadratic 2-D dispersion), and then again a $\mathrm{V}$-shape, but with approximately twice the slope as around $\mathrm{E}_{\mathrm{D}}$. Broken translational symmetry due to the interaction with the substrate will induce a gap at $\mathrm{E}_{\mathrm{D}}$ and Rashba-like shifts, which will open a gap around $\mathrm{E}_{\mathrm{D}}$ and cause square-root singularities at the band edges.

The calculated DOS on a fine k-point mesh $\left(\Delta k \sim 5 \times 10^{4} \AA^{-1}\right.$; with $\sim 150,000$ eigenvalues in the energy range shown, with Fermi broadening of each eigenvalue) for the laterally shifted graphene at a separation of $3.875 \AA$ are shown in Fig. S5. The expected characteristics of the DOS are evident, including the gap and the jumps corresponding to the band edges. However, because the bands are not completely symmetric about $\mathrm{E}_{\mathrm{D}}$, there is an asymmetry in 
the DOS. Despite the fine sampling of k-space used, this sampling is not able to resolve the expected singularities at the jumps corresponding to the Rashba shifts. (The singularities at edges of the gap near $\mathrm{E}_{\mathrm{D}}$ are evident for the smallest broadenings.) To address this issue, the bands around the band edges were fitted and then the DOS was obtained analytically (cyan curves in the right and left insets), and then convoluted with a Gaussian (magenta curves) to approximate the expected line shapes. The predicted effects of the transferred spin-orbit coupling on the DOS of graphene give rise to distinctive features compared to the normal (non-relativistic) DOS.

\section{Van Hove singularity in graphene- $\mathrm{Bi}_{2} \mathrm{Se}_{3}$ bands}

The $\mathrm{dI} / \mathrm{dV}$ spectrum in Fig. 4b exhibit two prominent peaks at $\mathrm{E}_{1} \approx 60 \mathrm{meV}$ and $\mathrm{E}_{2} \approx 160$ $\mathrm{meV}$, respectively, which arises from van Hove singularities in the density of states (DOS) near critical points of the graphene- $\mathrm{Bi}_{2} \mathrm{Se}_{3}$ bands. Both peaks follow a $1 / \sqrt{E}$ behavior, characteristic of the Van Hove singularity in 1D parabolic dispersion. The observation of this singularity is reminiscent of the distinct feature of a spinsplit band in a two-dimensional electron gas. ${ }^{2,3}$ The appearance of the asymmetric peaks is the result of finite lifetime and experimental broadening. With Gaussian broadening, a near perfect fit, Fig. S6, is found for the peak at $E_{1}=62$ $\mathrm{meV}$, while a slight deviation is seen at higher energy for the peak at $\mathrm{E}_{2}=157 \mathrm{meV}$.
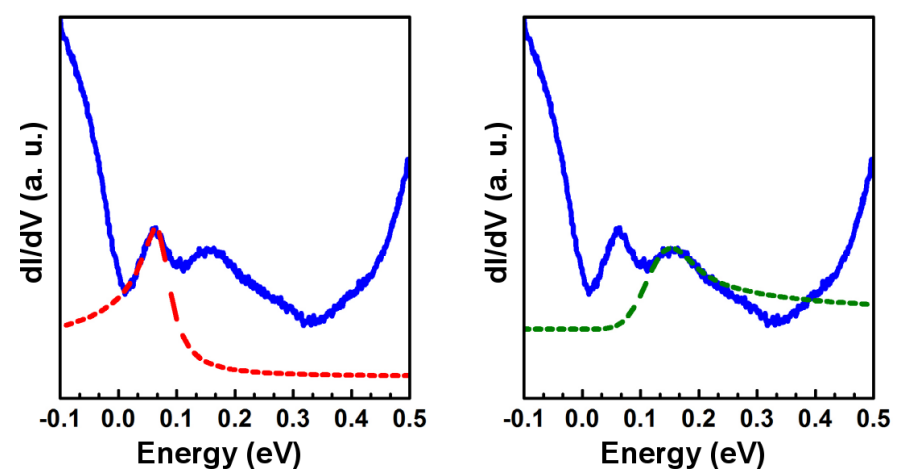

Figure S6: Fits of the two peaks in the dI/dV spectra with a Rashba-like 2D DOS, $n(E)=$ $B / \sqrt{\left|E-E_{i}\right|}$, and then convolved with a Gaussian, $f(E)=\frac{1}{\sqrt{2 \pi} \sigma} \exp \left(-\frac{1}{2}\left(\frac{x}{\sigma}\right)^{2}\right)$. Note that $E<E_{1}$ and $E>E_{2}$ before convoluting. For the fits of both peaks, $\mathrm{B}=0.055$, and $\sigma=22 \mathrm{meV}$, and $\mathrm{E}_{1}=62 \mathrm{meV}$ and $\mathrm{E}_{2}=157 \mathrm{meV}$.

Acknowledgement Supported by U.S. Department of Energy, Office of Basic Energy Sciences, Division of Materials Sciences and Engineering under Award DE-FG02-05ER46228.

\section{References}

1. Benia, H. M.; Lin, C.; Kern, K.; Ast, C. R. Reactive Chemical Doping of the $\mathrm{Bi}_{2} \mathrm{Se}_{3}$ Topological Insulator. Phys. Rev. Lett. 2011, 10\%, 177602.

2. Ast, C. R.; Wittich, G.; Wahl, P.; Vogelgesang, R.; Pacilé, D.; Falub, M. C.; Moreschini, L.; Papagno, M.; Grioni, M.; Kern, K. Local detection of spin-orbit splitting by scanning tunneling spectroscopy. Phys. Rev. B 2007, 75, 201401.

3. Ast, C. R. Extracting the Rashba splitting from scanning tunneling microscopy measurements. J. Electron Spectrosc. Relat. Phenom. 2015, 201, 30-35. 